\title{
Flat-type colorectal advanced adenomas (laterally spreading tumors) have different genetic and epigenetic alterations from protruded-type advanced adenomas
}

\author{
Taiga Takahashi ${ }^{1}$, Katsuhiko Nosho ${ }^{1}$, Hiroyuki Yamamoto ${ }^{1}$, Masashi Mikami ${ }^{1}$, \\ Hiroaki Taniguchi ${ }^{1}$, Nobuki Miyamoto ${ }^{1}$, Yasushi Adachi ${ }^{1}$, Fumio Itoh ${ }^{2}$, Kohzoh Imai $^{3}$ \\ and Yasuhisa Shinomura ${ }^{1}$ \\ ${ }^{1}$ First Department of Internal Medicine, Sapporo Medical University School of Medicine, Sapporo, Japan; \\ ${ }^{2}$ Division of Gastroenterology and Hepatology, Department of Internal Medicine, St Marianna University \\ School of Medicine, Kawasaki, Japan and ${ }^{3}$ Sapporo Medical University, Sapporo, Japan
}

\begin{abstract}
Morphologically, colorectal adenomas can be divided into two groups, protruded-type and flat-type. However, the accurate frequencies of genetic and epigenetic alterations in flat-type colorectal advanced adenomas (laterally spreading tumors) have remained largely unknown. In the current study, we investigated genetic and epigenetic alterations in 101 flat-type colorectal advanced adenomas and 68 protruded-type colorectal advanced adenomas by using direct DNA sequencing and quantitative real-time PCR (MethyLight), respectively. KRAS mutation was detected in a significantly higher percentage of flat-type adenomas $(35 \%)$ than in protruded-type adenomas (13\%). When the samples were limited to the tumors in the distal colon, the difference of KRAS mutation was still significant. KRAS mutation in G-to-A transitions at codons 12 and 13 was detected in a significantly higher percentage of flat-type adenomas (26\%) than in protruded-type adenomas (9\%). BRAF and $\beta$-catenin mutations were detected in 3 and $8 \%$ of the 101 flat-type adenomas, respectively. No significant difference was found between frequencies of those mutations in flat-type adenomas and protruded-type adenomas. Methylations of MGMT, CDKN2A (p16) and MLH1 were detected in 28, 33 and 9\% of the 101 flat-type adenomas, respectively. CDKN2A methylation was detected in a significantly lower percentage of flat-type adenomas than in protruded-type adenomas $(63 \%)$. Methylation of at least one gene was detected in a significantly lower percentage of flat-type adenomas $(54 \%)$ than in protruded-type adenomas (78\%). In conclusion, KRAS mutation was frequently detected in flat-type advanced adenomas and the mutational patterns in most of them with KRAS mutations were a transition from G-to-A. Therefore, these genetic alterations seem to play an important role in the development of flat-type advanced adenomas, especially in the distal colon. Epigenetic alterations infrequently occurred in flat-type advanced adenomas, suggesting that they have different genetic and epigenetic alterations from those of protruded-type advanced adenomas.

Modern Pathology (2007) 20, 139-147. doi:10.1038/modpathol.3800722; published online 24 November 2006
\end{abstract}

Keywords: flat-type colorectal adenoma; laterally spreading tumor; MethyLight; advanced adenoma; colorectal cancer; KRAS

Morphologically, colorectal adenomas can be divided into two groups, protruded-type and flat-type. As early detection of flat-type adenomas is difficult compared with protruded-type adenomas, they are sometimes overlooked and tend to be found at a late stage. ${ }^{1}$ Flat-type colorectal adenomas may be endo-

Correspondence: Dr K Nosho, MD, PhD, First Department of Internal Medicine, Sapporo Medical University, S.-1, W.-16, Chuo-ku, Sapporo 060-8543, Japan.

E-mail: nosho@sapmed.ac.jp

Received 12 June 2006; revised and accepted 10 October 2006; published online 24 November 2006 scopically defined by a height of less than half of their diameter or histologically defined by thickness of the lesion of less than twice that of the adjacent normal colonic mucosa. ${ }^{2}$ The Paris classification compares the height of the lesion to that of the height of closed cups of a biopsy forceps $(2.5 \mathrm{~mm}){ }^{3,4}$ Lesions protruding above the level of the closed jaws of the biopsy forceps are classified as protruded-type, whereas those that protrude below this level are classified as flat-type.

Large flat-type colorectal tumors are often labeled as 'carpet lesions' in the United States and 'laterally spreading tumors' in Japan. ${ }^{5-10}$ Laterally spreading 
tumors are superficial neoplasms that spread laterally over the mucosa, and these have recently received significant attention from gastroenterologists. However, the accurate frequencies of these genetic and epigenetic alterations in flat-type colorectal tumors have remained largely unknouwn.

Recently, we reported a case of laterally spreading tumor with interstitial deletion, including $\beta$-catenin exon $3 .{ }^{11}$ The $\beta$-catenin protein has two major functions. First, it acts as a cell-cell adhesion regulatory protein that binds cadherin. ${ }^{12}$ Second, it is thought to act as a downstream transcriptional activator in the Wnt signaling pathway. However, the frequency of $\beta$-catenin mutations in flat-type colorectal tumors has not been reported.

On the other hand, the RAS-RAF signaling pathway mediates cellular responses to growth signals not only in colorectal cancers but also in colorectal adenomas. KRAS mutation has been shown to be associated with epigenetic silencing of O6-methylguanine-DNA methyltransferase (MGMT), which is known to encode a DNA repair protein that removes potentially carcinogenic and cytotoxic alkyl adducts from the O6 position of guanine. ${ }^{13-17}$ Alterations in the MGMT gene impair the ability of the MGMT protein to remove alkyl groups from the $\mathrm{O} 6$ position of guanine. Therefore, alterations in the MGMT gene are thought to increase the mutational rate and the risk of cancer. ${ }^{13,16}$ Epigenetic silencing of MGMT has been shown to be associated with the appearance of G-to-A transitions in KRAS mutations during colorectal tumorigenesis. ${ }^{15,16}$ On the other hand, $B R A F$ mutations have also been reported in hyperplastic polyps and serrated adenomas. ${ }^{18}$ Subsequently, $B R A F$ mutations have been shown to be associated with the epigenetic silencing of MLH1 but not with germline mutation of mismatch repair genes. ${ }^{19}$ Regarding epigenetic alterations in flat-type colorectal tumors, Sakamoto et a ${ }^{20}$ reported that RASS$F 1 A$ promoter hypermethylation was detected in $81 \%$ of such tumors.

In the current study, we investigated genetic and epigenetic alterations in 101 flat-type colorectal advanced adenomas, 68 protruded-type colorectal advanced adenomas, and 35 colorectal cancers by using direct DNA sequencing and quantitative realtime PCR to measure DNA methylation (MethyLight), respectively. To our knowledge, this is the first report presenting results of genetic and epigenetic analysis of over 100 flat-type colorectal advanced adenoma tissues.

\section{Materials and methods}

\section{Patients and Tissue Samples}

Formalin-fixed paraffin-embedded tissues of 169 colorectal adenomas and 35 colorectal cancers were obtained from patients who had undergone polypectomy or surgical treatment. All of the adenoma
Table 1 Clinicopathological characteristics of colorectal advanced adenomas

\begin{tabular}{|c|c|c|}
\hline \multirow[t]{2}{*}{ Characteristics } & \multicolumn{2}{|c|}{ Morphology } \\
\hline & $\begin{array}{l}\text { Flat-type } \\
(\mathrm{n}=101)\end{array}$ & $\begin{array}{l}\text { Protruded-type } \\
\quad(\mathrm{n}=68)\end{array}$ \\
\hline Age (years \pm s.d.)* & $65.6 \pm 9.5$ & $62.1 \pm 9.4$ \\
\hline Mean size $(m m \pm$ s.d.) & $22.8 \pm 15.0$ & $20.5 \pm 12.0$ \\
\hline \multicolumn{3}{|l|}{ Gender** } \\
\hline Male & 59 & 52 \\
\hline Female & 42 & 16 \\
\hline \multicolumn{3}{|l|}{ Location $^{* * *}$} \\
\hline Proximal & 67 & 31 \\
\hline Distal & 34 & 37 \\
\hline \multicolumn{3}{|l|}{ Histopathology } \\
\hline Serrated adenoma & 3 & 1 \\
\hline $\begin{array}{l}\text { Adenoma with low-grade } \\
\text { dysplasia }\end{array}$ & 32 & 17 \\
\hline $\begin{array}{l}\text { Adenoma with high-grade } \\
\text { dysplasia }\end{array}$ & 13 & 16 \\
\hline $\begin{array}{l}\text { Intramucosal carcinoma and } \\
\text { carcinoma in situ }\end{array}$ & 53 & 34 \\
\hline
\end{tabular}

*Flat vs protruded, $P=0.0193$; **flat $v s$ protruded, $P=0.0153$; $* *$ flat vs protruded, $P=0.0074$.

samples were limited to advanced adenomas to match the histopathology of flat-type and protrudedtype adenomas. Advanced adenoma was defined as an adenoma of $10 \mathrm{~mm}$ or more in diameter, an adenoma with high-grade dysplasia. Intramucosal carcinoma and carcinoma in situ were classified as adenoma with high-grade dysplasia. ${ }^{21,22}$ The subjects were classified according to the most advanced lesion identified. These advanced adenoma samples consisted of four serrated adenomas, 49 adenomas with low-grade dysplasia, 29 adenomas with highgrade dysplasia, and 87 intramucosal carcinomas and carcinoma in situ. None of the patients reported a family history of colorectal cancers in interviews.

Locations of the colorectal tumors were divided into proximal colon (cecum, ascending and transverse colon) and distal colon (descending and sigmoid colon and rectum). Macroscopic types were divided into protruded-type (height of tumor $\geq 2.5 \mathrm{~mm}$ ) and flat-type (height of tumor $<2.5 \mathrm{~mm}$ ). The clinicopathological characteristics of 101 flattype adenomas and 68 protruded-type adenomas are shown in Table 1. Informed consent was obtained from each subject and the institutional review committee approved this study.

\section{Detection of KRAS Codon 12 and Codon 13, BRAF Codon 600 and $\beta$-catenin Exon 3 Mutations}

Mutations of KRAS (codon 12 and codon 13), BRAF (codon 600) and $\beta$-catenin exon 3 were detected by direct DNA sequencing. KRAS was amplified by 
PCR using primer pair: forward, $5^{\prime}$-AAAATGACTGA ATATAAACTTGTGG- $3^{\prime}$ and reverse, $5^{\prime}$-CTCTATTG TTGGATCATATTCGTC- $3^{\prime}$. BRAF was amplified by PCR using primer pair: forward, $5^{\prime}$-CTTCATGAAGA CCTCACAGT- $3^{\prime}$ and reverse, 5'-CATCCACAAAATG GATCCAG-3'. $\beta$-catenin exon 3 was amplified by PCR using primer pair: forward, $5^{\prime}$-GAACCAGACAG AAAAGCGGCTG- $3^{\prime}$ and reverse, $5^{\prime}$-ACTCATACAG GACTTGGGAGG-3'. Products were purified and then sequenced in both directions using Big Dye Terminator Cycle Sequencing kit (Applied Biosystems, Foster City, CA, USA). The sequence reactions were run and analyzed on an ABI 3100 Genetic Analyzer (Applied Biosystems).

\section{Quantitative Real-Time PCR to Measure DNA Methylation (MethyLight)}

Sodium bisulfite conversion of genomic DNA was performed as described previously. ${ }^{23,24}$ Quantitative real-time PCR to measure DNA methylation (MethyLight) was performed as previously. ${ }^{23}$ We used an ABI 7000 (Applied Biosystems, Foster City, CA, USA) for quantitative real-time PCR (Figure 1). Primers and probes specific for methylated DNA and used for MethyLight reactions are listed in Table 2. The percentage of methylated reference

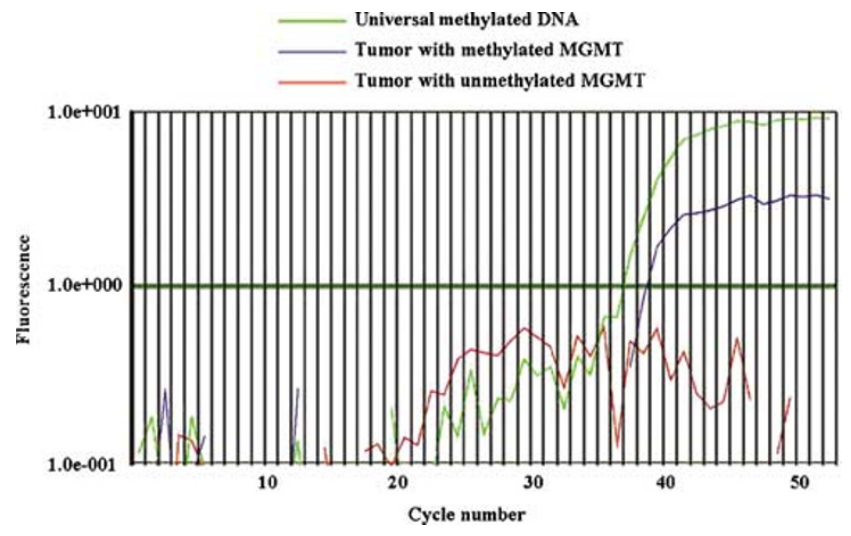

Figure 1 A quantitative real-time PCR to measure DNA methylation (MethyLight) showing the analysis of MGMT methylation. Bisulfite-converted universal methylated DNA was used for PMR calculation. The fluorescence is plotted vs the PCR cycle number for reaction and each sample is indicated.
(PMR) at a specific locus was calculated by dividing the GENE: ACTB ratio of a sample by the GENE: $A C T B$ ratio of Universal Methylated DNA (fully methylated) (Chemicon International, Temecula, CA, USA) and multiplying by 100 . We used PMR cutoff of four to distinguish methylation positive $(\mathrm{PMR}>4)$ from negative (PMR $\leq 4)$. The PMR cutoff of 4 was previously validated. ${ }^{24}$

\section{Immunohistochemistry}

Immunohistochemistry with an anti-human MGMT mouse monoclonal antibody (MAB16200, 1:100 dilution; Chemicon, Temecula, CA, USA) was carried out as described previously. ${ }^{25}$ The sections were examined microscopically by two well-trained pathologists who were blinded to the clinicopathological characteristics. Normal-appearing epithelium and stromal cells in each section provided positive internal controls for binding of the primary antibody. The immunoreactivity of MGMT protein was evaluated semiquantitatively, and a positive reactivity of $<5 \%$ of nuclei of tumor cells was regarded as negative. ${ }^{26}$

\section{Statistical Analysis}

Alteration of each target gene was assessed for associations with clinicopathological characteristics using the following statistical tests: Mann-Whitney $U$-test for age and size, and the $\chi^{2}$ two-tailed test or Fisher's exact test for the remaining parameters.

\section{Results} Clinicopathological Characteristics of Flat-Type
Adenoma and Protruded-Type Adenoma Tissues

The mean age of flat-type adenomas was significantly larger that of protruded-type adenomas $(P=0.0193)$ (Table 1). Regarding gender, flat-type adenomas were more frequently found in females than were protruded-type adenomas $(P=0.0153)$. Regarding tumor location, flat-type adenomas were more frequently located in the proximal colon than were protruded-type adenomas $(P=0.0074)$.

Table 2 MethyLight primer and probe sequences

\begin{tabular}{lll}
\hline HUGO gene name & $\begin{array}{l}\text { Forward primer sequences }\left(5^{\prime}-3^{\prime}\right) \\
\text { Reverse primer sequences }\left(5^{\prime}-3^{\prime}\right)\end{array}$ & Probe sequences $\left(5^{\prime}-3^{\prime}\right)$ \\
\hline$M G M T$ & $\begin{array}{l}\text { GCGTTTCGACGTTCGTAGGT } \\
\text { CACTCTTCCGAAAACGAAACG }\end{array}$ & 6FAM-CGCAAACGATACGCACCGCGA-BHQ-1 \\
TGKN2A & $\begin{array}{l}\text { TAGATTTCGGTTGATTGGTT } \\
\text { AACAACGTCCGACCTCCT }\end{array}$ & 6FAM-ACCCGACCCCGAACCGCG-BHQ-1 \\
AGGAAGAGCGGATAGCGATTT & 6FAM-CCCGCTACCTAAAAAAATATACGCTTACGCG-BHQ-1 \\
& TCTCGTCCTAAAACG &
\end{tabular}




\section{KRAS Codon 12 and Codon 13 Mutations}

KRAS mutation was detected in a significantly higher percentage of flat-type adenoma tissues (35\% of 101 tumors) than in protruded-type adenoma tissues (13\% of 68 tumors; $P=0.0019$ ) (Table 3). When the samples were limited to the tumors in the distal colon, the difference of KRAS mutation was still significant $(P=0.0007)$. On the other hand, no significant difference was found in the proximal colon. KRAS codon 12 mutation, codon 13 mutation and both mutations were detected in 20, 14 and 1 of the 35 flat-type adenomas, respectively (Figure 2a). In 15 of the 35 flat-type adenomas with $K R A S$ mutations, the mutational patterns were 13 GGC-toGAC (G13D). In 11 cases, they were 12 GGT-to-GAT (G12D). Various mutational patterns were found in the remaining cases: 12 GGT-to-GCT (G12A), 12 GGT-to-GTT (G12V), 12 GGT-to-AGT (G12S), and 12 GGT-to-TGT (G12C) (Table 4). KRAS mutation in

Table 3 Genetic and epigenetic alterations of colorectal advanced adenomas

\begin{tabular}{lccr}
\hline & \multicolumn{2}{c}{ Morphology } & P-value \\
\cline { 2 - 3 } & $\begin{array}{c}\text { Flat-type } \\
\mathrm{n}=101(\%)\end{array}$ & $\begin{array}{c}\text { Protruded-type } \\
\mathrm{n}=68(\%)\end{array}$ \\
\hline Mutation & & & \\
KRAS & $35(34.7)$ & $9(13.2)$ & 0.0019 \\
BRAF & $3(3.0)$ & $2(2.9)$ & 0.9999 \\
$\beta$-catenin & $8(7.9)$ & $4(5.9)$ & 0.6129 \\
Methylation & & & 0.2957 \\
MGMT & $28(27.7)$ & $24(35.3)$ & 0.0001 \\
CDKN2A & $33(32.7)$ & $43(63.2)$ & 0.1516 \\
MLH1 & $9(8.9)$ & $11(16.2)$ & \\
\hline
\end{tabular}

G-to-A transitions at codon 12 and codon 13 was detected in a significantly higher percentage of flattype adenoma tissues (26\% of 101 tumors) than in protruded-type adenoma tissues (9\% of 68 tumors; $P=0.0059)$. When the samples were limited to the tumors in the distal colon, the difference of KRAS mutation in G-to-A transitions was still significant $(P=0.0240)$.

In flat-type adenomas, KRAS mutation was not correlated significantly with any clinicopathological characteristics. On the other hand, in protrudedtype adenoma tissues, KRAS mutation was correlated significantly with size $(P=0.0003)$ and gender (female $>$ male) $(P=0.0011)$. On the other hand, KRAS mutation was detected in $13(37 \%)$ of the 35 colorectal cancer tissues. KRAS mutation in G-to-A transitions at codon 12 and codon 13 was detected in nine $(26 \%)$ of the 35 colorectal cancers.

\section{BRAF Codon 600 (V600E) Mutation}

$B R A F$ mutation was detected in three (3\%) of the 101 flat-type adenoma tissues. All of the tumors

Table 4 Spectrum of KRAS mutations in 101 flat-type colorectal advanced adenomas

\begin{tabular}{lc}
\hline Mutation (amino acid change) & $\begin{array}{c}\text { Number of FCAA } \\
\text { patients (\%) }\end{array}$ \\
\hline 12 GGT-to-GAT (G12D) & $10(9.9)$ \\
12 GGT-to-GCT (G12A) & $4(4.0)$ \\
12 GGT-to-GTT (G12V) & $4(4.0)$ \\
12 GGT-to-AGT (G12S) & $1(1.0)$ \\
12 GGT-to-TGT (G12C) & $1(1.0)$ \\
12 GGT-to-GAC (G13D) & $14(13.9)$ \\
GGC-to-GAC (G13D) & $1(1.0)$
\end{tabular}

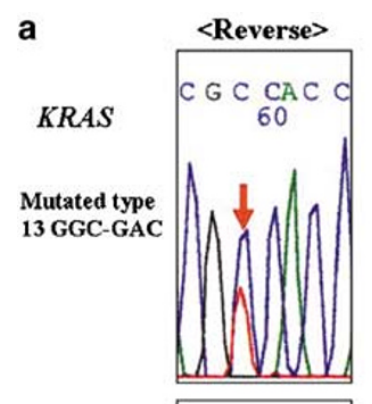

b

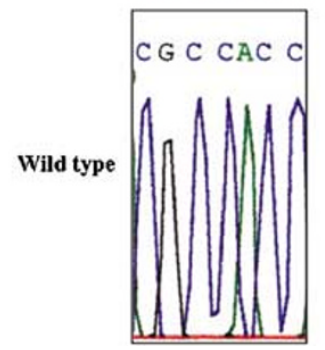

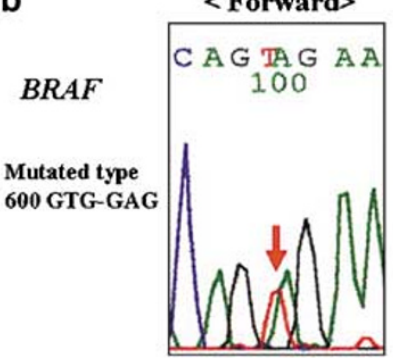

C

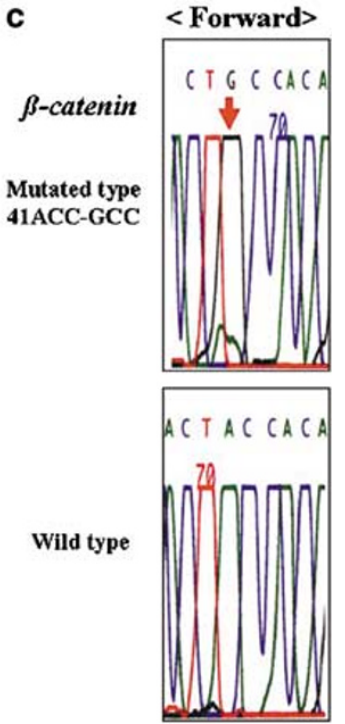

Figure 2 DNA direct sequencing of colorectal adenomas with $K R A S, B R A F$ or $\beta$-catenin exon 3 mutation. Wild-type, sequence of a wild case; mutated type, sequence of a mutated case. (a) A GGC-to-GAC change (G13D) at KRAS codon 13. (b) A GTG-to-GAG change (V600E) at BRAF codon 600. (c) An ACC-to-GCC (T41A) change at $\beta$-catenin codon 41. 
demonstrated missense mutations at codon 600 (V600E) (Figure 2b). There was a mutually exclusive relationship between KRAS and BRAF mutations. In flat-type adenoma tissues, $B R A F$ mutation was detected in two $(67 \%)$ of the three serrated adenomas. No significant difference was found between frequencies of those mutations in flat-type adenomas and protruded-type adenomas (Table 3). In flattype adenoma and protruded-type adenoma tissues, $B R A F$ mutation was not correlated significantly with any clinicopathological characteristics. $B R A F$ mutation was detected in three $(9 \%)$ of the 35 colorectal cancer tissues.

\section{$\beta$-catenin Exon 3 Mutation}

$\beta$-catenin exon 3 mutation was detected in eight (8\%) of the 101 flat-type adenoma tissues. These mutations were single-base substitutions that were located within the critical serine/threonine codons for GSK-3 $\beta$ phosphorylation of $\beta$-catenin (codons 29-48) (Figure 2c). All sites of $\beta$-catenin exon 3 missense mutation have been reported previously. ${ }^{27-31}$ Interstitial deletions of $\beta$-catenin exon 3 were detected in two flat-type adenoma tissues. No significant difference was found between frequencies of those mutations in flat-type adenomas and protruded-type adenomas (Table 3). In flattype adenoma and protruded-type adenoma tissues, $\beta$-catenin exon 3 mutation was not correlated significantly with any clinicopathological characteristics. On the other hand, $\beta$-catenin exon 3 mutation was detected in three $(9 \%)$ of the 35 colorectal cancer tissues.

\section{Methylation of MGMT, CDKN2A (p16) and MLH1}

The frequencies of methylation of MGMT, CDKN2A and $M L H 1$ in flat-type adenoma and protruded-type adenoma tissues are summarized in Table 3. Methylations of MGMT, CDKN2A and MLH1 were detected in $28(28 \%), 33(33 \%)$ and $9(9 \%)$ of the 101 flat-type adenoma tissues, respectively.

CDKN2A methylation was detected in a significantly lower percentage of flat-type adenoma tissues than in protruded-type adenoma tissues (63\% of 68 tumors; $P<0.0001$ ). When the samples were limited to the tumors in the proximal colon and the distal colon, respectively, the difference of CDKN2A methylation was still significant (data not shown). On the other hand, a significant difference was not found between frequencies of any other methylations in flat-type adenoma and protrudedtype adenoma tissues. Methylation of at least one gene was detected in a significantly lower percentage $(P=0.0012)$ of flat-type adenoma tissues $(54 \%$ of 101 tumors) than in protruded-type adenoma tissues (78\% of 68 tumors). When the samples were limited to the tumors in the proximal colon and the distal colon, respectively, the differences were still significant (data not shown).
In flat-type adenoma tissues, MGMT methylation was correlated significantly with age $(P=0.0316)$ and location (distal $>$ proximal) $(P=0.0087)$. On the other hand, in protruded-type adenoma tissues, CDKN2A was correlated significantly with size $(P=0.0088)$ and $M G M T$ methylation was correlated significantly with age $(P=0.0178)$ and location (distal $>$ proximal) $(P=0.0025)$. Although $M G M T$ methylation was detected in a higher percentage of flat-type adenomas with KRAS mutation in G-to-A transition, no significant relationship was found between the mutation and the methylation. On the other hand, methylation of MGMT, CDKN2A and MLH1 was detected in $11(31 \%), 14(40 \%)$ and 4 $(11 \%)$ of the 35 colorectal cancers, respectively.

\section{Immunohistochemical Staining of MGMT in Flat-Type Adenomas with or without MGMT Methylation}

The expression of MGMT was analyzed immunohistochemically in 30 flat-type adenoma tissues (Figure 3). Expression of MGMT protein was negative in six $(20 \%)$ of the 30 tumors. Expression of MGMT protein was negative in four $(40 \%)$ of the 10 tumors with MGMT methylation and in two $(10 \%)$ of the 20 tumors without MGMT methylation (Figure 2a and b). Although MGMT methylation was detected in a higher percentage of flat-type adenomas with reduced protein expression, no significant correlation was found between them.

We further examined the expression of MGMT in 30 protruded-type adenomas and 20 colorectal cancers by immunohistochemistry. MGMT expression was negative in $27 \%$ of protruded-type adenomas and $25 \%$ of colorectal cancers. No significant difference was found between frequencies of the MGMT negativity in adenomas (flat-type and protruded-type) and colorectal cancers.

\section{Discussion}

In the current study, KRAS mutation was detected in a significantly higher percentage of flat-type advanced adenomas than in protruded-type advanced adenomas. The mutational patterns in most of the flat-type adenomas with KRAS mutations were a transition from G-to-A. Mutations of $B R A F$ and $\beta$-catenin were detected in some flat-type adenomas. On the other hand, methylation of at least one gene was detected in a significantly lower percentage of flat-type adenomas than in protruded-type adenomas. These results suggest flat-type advanced adenomas have distinct genetic and epigenetic features different from those of protruded-type advanced adenomas (Figure 4).

Although more than 10 years have passed since $\mathrm{Kudo}^{6}$ advocated the new category 'laterally spreading tumor', only a few genetic alterations of laterally spreading tumors such as KRAS, $\beta$-catenin and $p 53$ mutations, ${ }^{5,8,10,11}$ and overexpression of $\mathrm{COX}-2^{7}$ and 

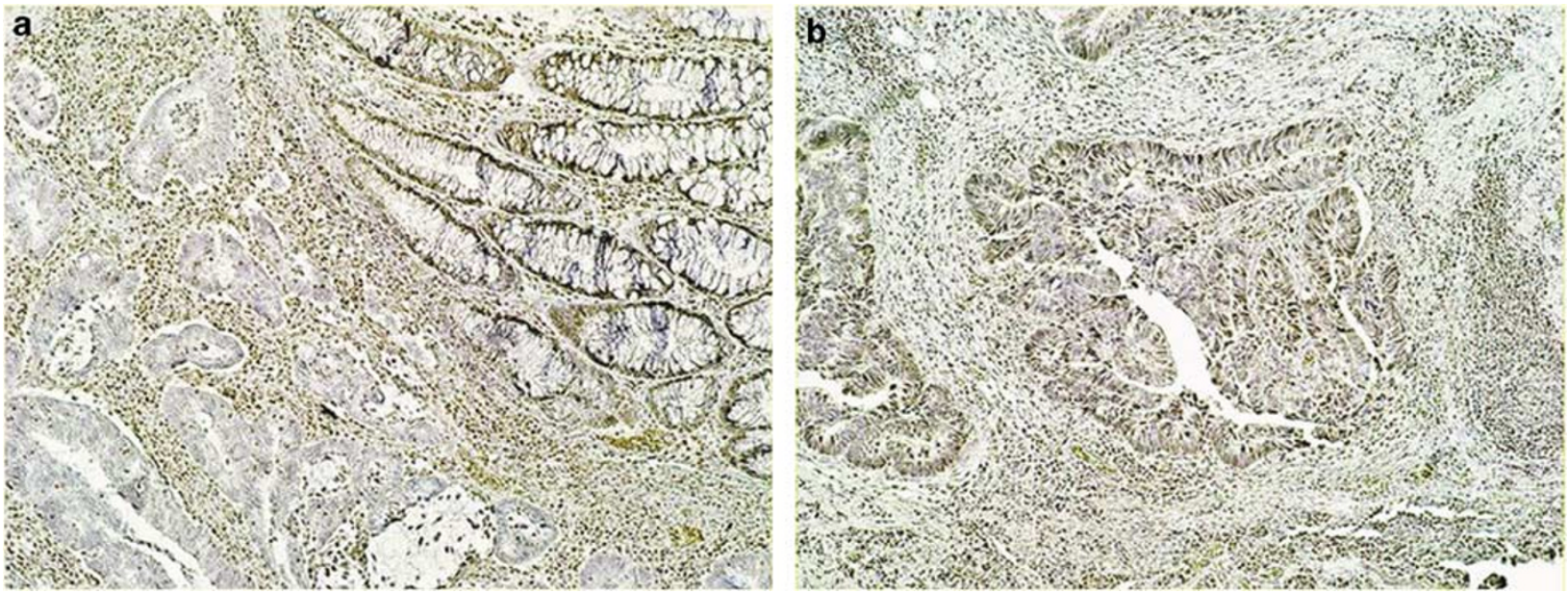

Figure 3 Immunohistochemical staining of MGMT protein in colorectal adenomas. (a) A tumor with MGMT methylation showed no staining in tumor cells but clear staining in the nuclei of non-tumor cells $(\times 100)$. (b) A tumor without MGMT methylation showed staining for the MGMT protein in tumor cell nuclei $(\times 100)$.

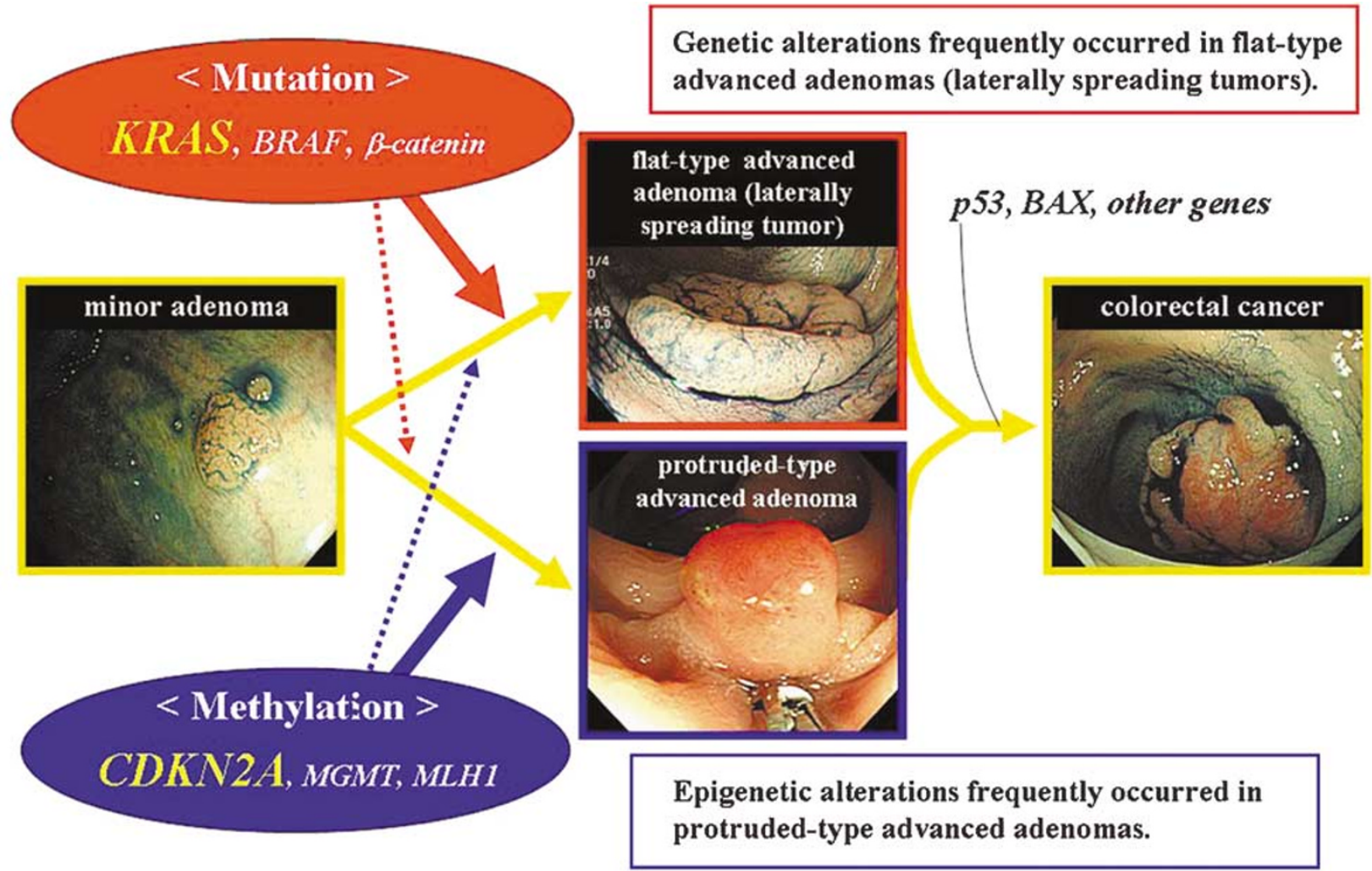

Figure 4 A diagram to illustrate the genetic pathways of flat-type advanced adenoma (laterally spreading tumor) and protruded-type advanced adenoma leading to colorectal cancers. Flat-type advanced adenomas seems to have different genetic and epigenetic alterations from those of protruded-type advanced adenomas.

gastrin protein ${ }^{8}$ have been reported. laterally spreading tumors are defined as lesions $>10 \mathrm{~mm}$ in diameter with a low vertical axis that extend laterally along the luminal wall, ${ }^{6}$ and most of them remain as adenoma or early invasive cancer. ${ }^{7}$ As the criteria of laterally spreading tumor are almost consistent with those of flat-type advanced adeno- ma, we compared the frequencies of KRAS mutations in flat-type advanced adenomas in the current study and in laterally spreading tumors in previous studies.

Kusaka et $a l^{10}$ reported that KRAS codon 12 mutation was detected in nine (50\%) of 18 laterally spreading tumor tissues. With one exception, these 
tumors had the same mutational pattern: 12 GGT-toGTT $(\mathrm{G} 12 \mathrm{~V})$. Mukawa et $a l^{8}$ reported that KRAS mutation was detected in $18(50 \%)$ of 36 laterally spreading tumor tissues and in 13 (50\%) of 26 protruded-type tumor tissues. In eight of the 21 tumors with KRAS mutations, the mutational patterns were 12 GGT-to-GTT (G12V). In further eight cases, they were 12 GGT-to-GAT (G12D). Noro et $a l^{5}$ reported that KRAS mutation was detected in six (21\%) of 28 laterally spreading tumor tissues and in six $(26 \%)$ of 23 protruded-type tumor tissues. Frequent mutational patterns were 12 GGT-to-TGT (G12C) in laterally spreading tumors and 12 GGT-toGAT (G12D) in protruded-type tumors. No KRAS mutations within codon 13 were detected in any of the tumors.

The discrepancy in these mutational patterns may be because of the small numbers of samples analyzed in previous studies and/or differences in the methods of measurement. On the other hand, KRAS mutation was detected in a smaller percentage of flat-type adenomas in the current study than in laterally spreading tumors in previous studies. The reason for the low frequency of KRAS mutations in flat-type adenoma tissues is not known. It might be due to the fact that depressed areas were seen in the surfaces of some flat-type adenoma tissues. Umetani et $a l^{32}$ reported that the frequency of KRAS mutations in flat-type tumors with depressed areas was significantly lower than that in flat-type tumors without depressed areas.

In the current study, when the samples were limited to the tumors in the distal colon, KRAS mutation in G-to-A transitions was detected in a significantly higher percentage of flat-type adenomas than in protruded-type adenomas. This result suggests that these genetic alterations play an important role in the development and/or progression of flat-type advanced adenomas (laterally spreading tumors), especially in the distal colon. In addition, Nagasaka et $a 1^{13}$ reported that $K R A S$ mutation in G-to-A transitions at codon 12 and codon 13 was detected in 51 (22\%) of 234 colorectal cancers. This is consistent with our data on mutational patterns in flat-type advanced adenomas and colorectal cancers. Therefore, colorectal cancers with KRAS mutation in G-to-A transitions seem to largely arise from flat-type advanced adenomas. Further analysis is needed to clarify this issue.

Promoter hypermethylation of CpG islands provides an epigenetic mechanism for transcriptional repression of genes such as MGMT, CDKN2A and MLH1. ${ }^{13-16,18}$ In addition, aberrant methylation of these genes in association with other genetic alterations has been shown to be an important mechanism of colorectal carcinogenesis. ${ }^{13,15,16,18,19}$ Petko et al ${ }^{14}$ found by using methylation-specific polymerase chain reaction (MSP) that methylation of $M G M T$, CDKN2A and MLH1 occurred in 49, 34 and $7 \%$ of adenomas, respectively, and that they are more common in histopathologically advanced adeno- mas. The frequencies of methylation of CDKN2A (45\%; 76/169) and MLH1 (12\%; 20/169) in advanced adenomas in the current study are similar to these previously reported frequencies. However, MGMT methylation was detected in a smaller percentage of advanced adenomas $(31 \% ; 52 / 169)$ and colorectal cancers $(31 \% ; 11 / 35)$ in the current study than in colorectal adenomas in that previous study. ${ }^{14}$ The discrepancy may be because of the differences in the methods of measurement using MethyLight and MSP.

In the current study, not only CDKN2A methylation but also methylation of at least one gene was detected in a significantly lower percentage of flattype adenomas than in protruded-type adenomas. Therefore, epigenetic alterations might infrequently occur in flat-type adenomas.

MGMT methylation was not correlated significantly with KRAS mutation in G-to-A transition in flat-type adenoma tissues, despite their high rates of KRAS mutations. Therefore, some other mismatch repair genes for KRAS mutation might contribute to the mutational patterns, which were a transition from G-to-A, in flat-type adenoma tissues. In addition, no significant correlation was found between the presence of $M G M T$ methylation and reduced MGMT protein expression. Six tumors with MGMT methylation expressed MGMT protein. This might be due to a low level of methylation, which reflects methylation of only a few tumor cells, or methylation of one allele but absence of methylation of the other allele of the gene. On the other hand, expression of MGMT protein was reduced in two tumors without MGMT methylation. It has been reported that $M G M T$ mutations are infrequently found in colorectal cancers and contribute to reduction of their protein function. ${ }^{17}$ Therefore, in these cases, reduced expression of MGMT protein might have arisen from genetic and/or other epigenetic abnormalities.

In flat-type adenoma tissues, $B R A F$ mutation was frequently detected in serrated adenomas. Therefore, $B R A F$ mutations are thought to be associated with the hyperplastic polyp-serrated adenoma-carcinoma pathway not only in protruded-type adenomas but also in flat-type adenomas. $\beta$-catenin exon 3 mutation was detected in $8 \%$ of the flat-type adenomas in the current study. As $\beta$-catenin is involved in cell adhesion, abnormalities of $\beta$-catenin might play an important role not only in the tumorigenesis but also in the morphological features of a subset of flat-type adenomas (laterally spreading tumors). ${ }^{11,12}$

In summary, KRAS mutation was frequently detected in flat-type advanced adenoma tissues and the mutational patterns in most of them with KRAS mutations were a transition from G-to-A. Therefore, these genetic alterations seem to play an important role in the development of flat-type advanced adenomas (laterally spreading tumors), especially in the distal colon. Epigenetic alterations infrequently occurred in flat-type advanced 
adenomas, suggesting that they have different genetic and epigenetic alterations from those of protruded-type advanced adenomas.

\section{Acknowledgements}

This study was supported by Grants-in-Aid for Scientific Research from the Ministry of Education, Culture, Sports, Science and Technology of Japan (HY and KI) and Grants-in-Aid for Cancer Research and for the Third Term Comprehensive 10-Year Strategy for Cancer Control from the Ministry of Health, Labor and Welfare of Japan (HY and KI).

\section{Ethics approval statement}

An informed consent was obtained from each patient and the institutional review committee approved this study.

\section{Conflict of interest statement}

None decleared.

\section{References}

1 Kuramoto S, Oohara T. Flat early cancers of the large intestine. Cancer 1989;64:950-955.

2 Tsuda S, Veress B, Toth E, et al. Flat and depressed colorectal tumours in a southern Swedish population: a prospective chromoendoscopic and histopathological study. Gut 2002;51:550-555.

3 The Paris endoscopic classification of superficial neoplastic lesions: esophagus, stomach, and colon. Gastrointest Endosc 2003;58:S3-S43.

4 Soetikno R, Friedland S, Kaltenbach T, et al. Nonpolypoid (flat and depressed) colorectal neoplasms. Gastroenterology 2006;130:566-576.

5 Noro A, Sugai T, Habano W. Analysis of Ki-ras and p53 gene mutations in laterally spreading tumors of the colorectum. Pathol Int 2003;53:828-836.

6 Kudo S. Endoscopic mucosal resection of flat and depressed types of early colorectal cancer. Endoscopy 1993;25:455-461.

7 Yamashita K, Arimura Y, Shimizu H, et al. Increased cyclooxygenase-2 expression in large flat colorectal tumors (laterally spreading tumors). J Gastroenterol 2003;38:69-73.

8 Mukawa K, Fujii S, Takeda J, et al. Analysis of K-ras mutations and expression of cyclooxygenase-2 and gastrin protein in laterally spreading tumors. J Gastroentrol Hepatol 2005;20:1584-1590.

9 Japanese Society for Cancer of the Colon and Rectum. Japanese Classification of Colorectal Carcinoma. Kanehara: Tokyo, Japan, 1997.

10 Kusaka T, Fukui H, Sano Y, et al. Analysis of K-ras codon 12 mutations and p53 overexpression in colorectal nodule-aggregating tumors. J Gastroenterol Hapatol 2001;15:1151-1157.

11 Nosho K, Yamamoto H, Mikami M, et al. Laterally spreading tumour in which interstitial deletion of betacatenin exon 3 was detected. Gut 2005;54:1504-1505.
12 Wong NA, Pignatelli M. Beta-catenin-a linchpin in colorectal carcinogenesis? Am J Pathol 2002;160: 389-401.

13 Nagasaka T, Sasamoto H, Notohara K, et al. Colorectal cancer with mutation in BRAF, KRAS, and wild-type with respect to both oncogenes showing different patterns of DNA methylation. J Clin Oncol 2004;22: 4584-4594.

14 Petko Z, Ghiassi M, Shuber A, et al. Aberrantly methylated CDKN2A, MGMT, and MLH1 in colon polyps and in fecal DNA from patients with colorectal polyps. Clin Cancer Res 2005;11:1203-1209.

15 Shen L, Kondo Y, Rosner GL, et al. MGMT promoter methylation and field defect in sporadic colorectal cancer. J Natl Cancer Inst 2005;97:1330-1338.

16 Esteller M, Toyota M, Sanchez-Cespedes M, et al. Inactivation of the DNA repair gene O6-methylguanine-DNA methyltransferase by promoter hypermethylation is associated with $\mathrm{G}$ to $\mathrm{A}$ mutations in K-ras in colorectal tumorigenesis. Cancer Res 2000;60: 2368-2371.

17 Halford S, Rowan A, Sawyer E, et al. O(6)-methylguanine methyltransferase in colorectal cancers: detection of mutations, loss of expression, and weak association with G:C>A:T transitions. Gut 2005;54:797-802.

18 Kambara T, Simms LA, Whitehall VL, et al. BRAF mutation is associated with DNA methylation in serrated polyps and cancers of the colorectum. Gut 2004;53:1137-1144.

19 Domingo E, Espin E, Armengol M, et al. Activated BRAF targets proximal colon tumors with mismatch repair deficiency and MLH1 inactivation. Genes Chromosomes Cancer 2005;39:138-142.

20 Sakamoto N, Terai T, Ajioka Y, et al. Frequent hypermethylation of RASSF1A in early flat-type colorectal tumors. Oncogene 2004;23:8900-8907.

21 Imperiale TF, Ransohoff DF, Itzkowitz SH, et al. Fecal DNA vs fecal occult blood for colorectal-cancer screening in an average-risk population. $\mathrm{N}$ Engl J Med 2004;351:2704-2714.

22 Morikawa T, Kato J, Yamaji Y, et al. A comparison of the immunochemical fecal occult blood test and total colonoscopy in the asymptomatic population. Gastroenterology 2005;129:422-428.

23 Eads CA, Danenberg KD, Kawakami K, et al. MethyLight: a high-throughput assay to measure DNA methylation. Nucleic Acids Res 2000;28:E32.

24 Ogino S, Brahmandam M, Kawasaki T, et al. Epigenetic profiling of synchronous colorectal neoplasias by quantitative DNA methylation analysis. Mod Pathol 2006;19:1083-1090.

25 Nosho K, Yoshida M, Yamamoto H, et al. Association of Ets-related transcriptional factor E1AF expression with overexpression of matrix metalloproteinases, COX-2 and iNOS in the early stage of colorectal carcinogenesis. Carcinogenesis 2005;26:892-899.

26 Park TJ, Han SU, Cho YK, et al. Methylation of O(6)methylguanine-DNA methyltransferase gene is associated significantly with K-ras mutation, lymph node invasion, tumor staging, and disease free survival in patients with gastric carcinoma. Cancer 2001;92: 2760-2768.

27 Mirabelli-Primdahl L, Gryfe R, Kim H, et al. Betacatenin mutations are specific for colorectal carcinomas with microsatellite instability but occur in endometrial carcinomas irrespective of mutator pathway. Cancer Res 1999;59:3346-3351. 
28 Johnson V, Volikos E, Halford SE, et al. Exon 3 betacatenin mutations are specifically associated with colorectal carcinomas in hereditary non-polyposis colorectal cancer syndrome. Gut 2005;54:264-267.

29 Samowitz WS, Powers MD, Spirio LN, et al. Betacatenin mutations are more frequent in small colorectal adenomas than in larger adenomas and invasive carcinomas. Cancer Res 1999;59:1442-1444.

30 Miyaki M, Iijima T, Kimura J, et al. Frequent mutation of beta-catenin and APC genes in primary colorectal tumors from patients with hereditary nonpolyposis colorectal cancer. Cancer Res 1999;59: 4506-4509.

31 Shitoh K, Koinuma K, Furukawa T, et al. Mutation of beta-catenin does not coexist with K-ras mutation in colorectal tumorigenesis. Dig Dis Sci 2004;49: 1631-1633.

32 Umetani N, Sasaki S, Masaki T, et al. Involvement of APC and K-ras mutation in non-polypoid colorectal tumorigenesis. Br J Cancer 2000;82:9-15. 